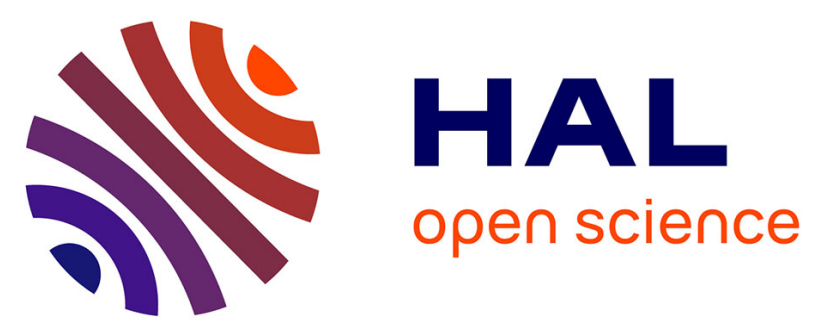

\title{
FURTHER RESULTS ON THE INITIATION AND GROWTH OF ADIABATIC SHEAR BANDS AT HIGH STRAIN RATES
}

\author{
T. Wright, R. Batra
}

\section{> To cite this version:}

T. Wright, R. Batra. FURTHER RESULTS ON THE INITIATION AND GROWTH OF ADIABATIC SHEAR BANDS AT HIGH STRAIN RATES. Journal de Physique Colloques, 1985, 46 (C5), pp.C5-323-C5-330. 10.1051/jphyscol:1985541 . jpa-00224772

HAL Id: jpa-00224772

https://hal.science/jpa-00224772

Submitted on 1 Jan 1985

HAL is a multi-disciplinary open access archive for the deposit and dissemination of scientific research documents, whether they are published or not. The documents may come from teaching and research institutions in France or abroad, or from public or private research centers.
L'archive ouverte pluridisciplinaire HAL, est destinée au dépôt et à la diffusion de documents scientifiques de niveau recherche, publiés ou non, émanant des établissements d'enseignement et de recherche français ou étrangers, des laboratoires publics ou privés. 


\title{
FURTHER RESULTS ON THE INITIATION AND GROWTH OF ADIABATIC SHEAR
} BANDS AT HIGH STRAIN RATES

\author{
T.W. Wright and R.C. Batra \\ Ballistic Research Laboratory, Aberdeen Proving Ground, MD. 21005, U.S.A. \\ *University of Missouri-Rolia, Rolza, MO. 65401, U.S.A.
}

Résumé - Par un calcul aux éléments finis on a étudié la croissance de perturbations dans la déformation par cisaillement simple d'un solide thermovisco-plastique avec adoucissement. On a trouvë que les trēs petites perturbations croissent lentement au début même si le pic de contrainte de la courbe contrainte-déformation homogène a été franchi mais qu'une croissance explosive peut avoir lieu par la suite. Durant la phase de croissance lente, la contrainte suit de très près la réponse homogène mais dans la phase explosive la contrainte chute précipitamment. Les grandes perturbations croissent à une vitesse initiale plus importante et accêlèrent rapidement nême avant le pic de contrainte de la déformation homogène. L'introduction d'un effet plastique dipolaire semble être stabilisante.

Abstract - Growth of perturbations in the simple shearing deformation of a softening thermo-visco-plastic solid have been examined by means of a finite element technique. It has been found that very small perturbations grow slowly at first, even though the underlying homogeneous stress-strain response has passed peak stress, but that eventually explosive growth occurs. During the slow growth phase, the stress follows the homogeneous response quite closely, but in the explosive phase, the stress drops precipitously. Large perturbations grow at a larger injtial rate, and accelerate rapidly even before peak homogeneous stress. Inclusion of a dipolar plastic effect appears to be stabilizing.

\section{I - INTRODUCTION AND BASIC EQUATIONS}

Adiabatic shear banding is a localization phenomenon that occurs during high rate plastic deformation in many materials. Since the heat generated by plastic flow usually tends to lower the flow stress, the response curve for adiabatic homogeneous deformation will fall below the isothermal response. If this thermal softening is stronger than both work and rate hardening together, then eventually a peak stress occurs at a critical value of strain, followed by decreasing stress for further increments of strain. Such a situation is generally unstable and presents an opportunity for strain localization to occur. In recent years there has been considerable interest in developing a quantitative, dynamical theory of adiabatic shear banding. Much of the recent literature, as well as some of the pioneering works have been listed and discussed briefly by Clifton, et a $1 . / 1 \%$. In this paper we present further calculations of the kind given previously by Wright and Batra /2/. In particular, we show the effect of changing the constitutive rate response and the magnitude of the perturbation. We also examine the effect of adding a gradient or dipolar response to the constitutive equations, at least for early times.

In order to concentrate on fundamentals, consider one dimensional shearing of a block of material that lies between the boundaries $\bar{y}=+h$. The deformation is assumed to be given completely by horizontal shearing. Thus the velocity field may be written

$$
\dot{\bar{x}}=\bar{v}(\bar{y}, \bar{t}) ; \dot{\bar{y}}=0 ; \dot{z}=0 \text {. }
$$

As used in $/ 2 /$, the balance and constitutive laws for this case are given here in 
nondimensional form.

$\begin{array}{ll}\text { Momentum: } & s, y=\rho_{p} \dot{v} \\ \text { Energy: } & \dot{\theta}=k \theta, y y+s \dot{\gamma}_{p} \\ \text { Elastic Response: } & \dot{s}=\mu\left(v, y-\dot{\gamma}_{p}\right) \\ \text { Reference Plastic Response: } & \kappa=\left(1+\frac{\psi}{\psi_{0}}\right)^{n} \\ \text { Work Hardening: } & \dot{W}_{p}=k \dot{\psi}=s \dot{\gamma}_{p} \\ \text { Yield Surface: } & |s|=(1-a \theta)\left(I+b\left|\dot{\gamma}_{p}\right|\right)^{m_{k}}\end{array}$

Boundary conditions are $v(\underline{+1}, t)=\underline{+1}, \theta, y(\underline{+1}, t)=0$. In these equations the shear stress is $s$, mass density is $p$, particle velocity is $v$, temperature rise is $\theta$, thermal conductivity is $k$, plastic strain rate is $\dot{\gamma}_{p}$, shear modulus is $\mu$, work hard-

ening parameter is $k$, and plastic strain in a reference test is $\psi$. The comma denotes partial differentiation with respect to the spatial coordinate $y$, and the superimposed dot denotes partial differentiation with respect to time $t$. In the energy equation plastic working acts as a source term. A linear elastic response is assumed as wel1 as the additive decomposition of shear strain into elastic and plastic parts. The reference plastic response is intended to be simply a curve fit to a slow isothermal test. The work hardening parameter is assumed to depend only on the plastic work no matter what the rate of the test. Finally, the yield surface is taken to depend on plastic strain rate, as well as stress and temperature. When $(2)_{6}$ is solved for $\dot{\gamma}_{p}$ as a function of $s, \theta$, and $k$, this last condition is seen to be simply an overstress rule for plastic flow when the stress-temperature point lies outside the static yield surface. The form chosen here is similar to one due to Litonski /3/. Note that the thermat softening depends only linearly on temperature.

In (2) the nondimensional variables and parameters are related to their dimensional (barred) counterparts as follows:

$$
\begin{aligned}
& y=\bar{y} / \mathrm{h} \quad \mathrm{t}=\overline{\mathrm{t}}_{\mathrm{\gamma}} \quad \mathrm{v}=\overline{\mathrm{v}} / \mathrm{h} \dot{\gamma}_{0} \quad \mathrm{~s}=\overline{\mathrm{s}} / \mathrm{\kappa}_{0} \quad \theta=\left(\overline{\bar{\theta}} \bar{\rho} \mathrm{c}_{\nu}\right) / \kappa_{0} \\
& \kappa=\bar{\kappa} / \kappa_{0} \quad \gamma=\bar{\gamma} \quad \psi=\bar{\psi} \quad \rho=\left(\bar{\rho} h^{2} \dot{\gamma}_{0}^{2}\right) / \kappa_{0} \quad k=\bar{k} /\left(\bar{\rho} c_{\nu} \dot{\gamma}_{0} h^{2}\right) \\
& \mu=\bar{\mu} / \kappa_{0} \quad a=\left(\bar{a}_{\kappa_{0}}\right) /\left(\bar{\rho} c_{\nu}\right) \quad b=\bar{b} \dot{\gamma}_{0} \quad \dot{\gamma}_{p}=\dot{\gamma}_{p} / \dot{\gamma}_{0}
\end{aligned}
$$

where $\dot{\gamma}_{0}=\bar{v}(h, \bar{t}) / h$ is the average applied strain rate between $\bar{y}=t h, k_{0}$ is the initial yield stress in the reference test, $c_{v}$ is the specific heat at constant volume, and $\bar{a}, \bar{b}, \psi_{0}, m$ and $n$ are material constants.

\section{II - HOMOGENEOUS SOLUTIONS AND PERTURBATIONS}

If we set $v=y$ and assume that $s, \theta$, and $k$ are independent of $y$, then equations (2) reduce to a set of ordinary differential equations in time with $s, \theta$, and $k$ as dependent variables. For initial conditions we assume $s(0)=1, \theta(0)=0$, and $\kappa(0)=1$, so that yielding begins at the initial time. For the assumed visco/plastic flow law, the stress/strain response is a smooth curve, which rises at the elastic slope initially and then, as plastic flow increases and the temperature rises, the curve bends over, passes through a single maximum, and finally decreases with further increments of strain. This type of calculated behavior is well known in the literature, eg. $/ 4,5,6,7 /$. Figure 1 shows typical results. For curve $A$ the nondimensional parameters are

$$
\begin{array}{ll}
\rho=3.928 \times 10^{-5}, k=3.978 \times 10^{-3}, & a=0.4973, \quad \mu=240.3 \\
n=0.09, & \psi_{0}=0.017, \quad b=5 \times 10^{6}, m=0.025
\end{array}
$$


and for curve $B$ all the numbers are the same except $b=5 \times 10^{5}$ and $\mathrm{m}=0.02$. Also shown in the figure are the reference stress/strain curve $(a=0, b=0)$ and the isothermal response $(a=0)$ corresponding to $A$ (case $B$ is similar).

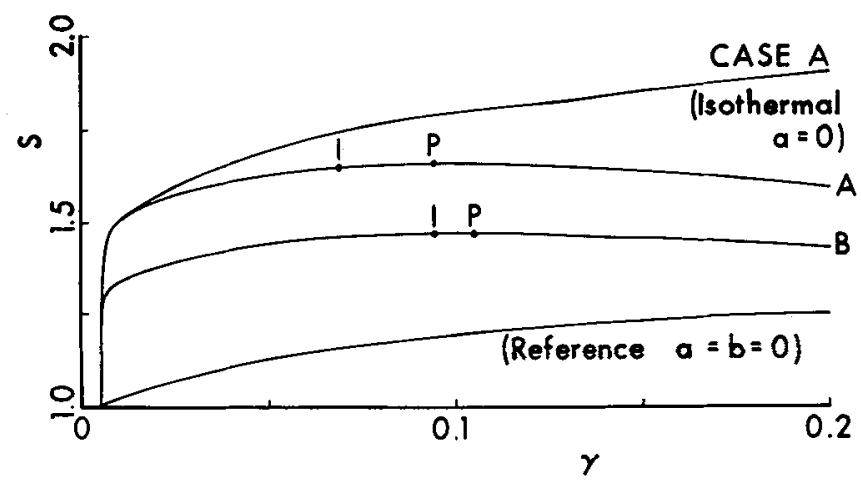

Fig. 1 - Homogeneous response curves. The reference curve, the isothermal curve for case $A$, and the adiabatic curves for both cases $A$ and $B$ are shown.

Perturbations to the homogeneous response have been introduced in the following manner. Just prior to the occurance of peak stress, the temperature was modified by adding a symmetric bump at the center. Two cases have been considered as shown ir: Figure 2, the larger perturbation being five times higher and broader than the smaller one, but similar in shape. With the new temperature distribution given, the stress was recalculated so that $(2)_{6}$ is still satisfied, all other variables being held fixed. Then the full problem using a 17 of (2) and the stated boundary conditions was restarted with the new field variables as initial data. After casting the equations into weak form, calculations were made using the finite element method, eg. see Becker; et a1. /8/.

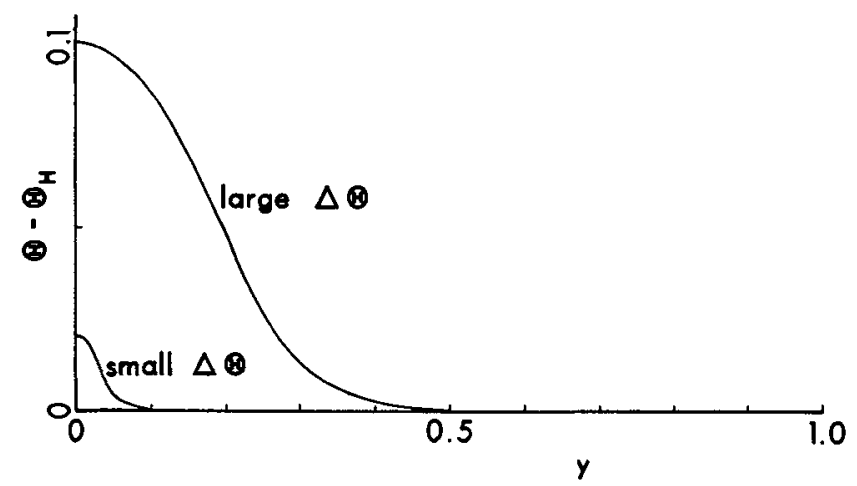

Fig 2. - Temperature perturbations added to the homogeneous response at $\mathrm{I}$.

Case $B$ with the smaller temperature bump as a perturbation was previously described in some detail in $/ 2 \%$. Figures 3 through 7 illustrate and compare the main features of the response for 277 cases considered to date. Because of the constant velocity maintained at the boundaries, average strain rate in the scaled variables for the strip $\gamma_{\text {ave }}$ is exactly equal to one. This fact allows direct comparisons between the homogeneous and inhomogeneous cases. Figure 3 shows the stress at the center of the strip as a function of average strain (or time). The perturbation was added at the point marked I, and the peak of the homogeneous response is marked P. For the small temperature bump the stress follows the homogeneous response until well past the point $P$, and then drops rapidiy as the shear band accelerates and localizes. The 
stress at other points of the strip is nearly the same, but lags slightly in time. Note that in real time (for $\dot{\gamma}_{0}=500 \mathrm{~s}^{-1}$, say) the stress collapse is delayed approximately $125 \mu$ s past peak stress $P$, and the rapid acceleration itself takes more than $20 \mu \mathrm{s}$. This behavior is entirely similar for both cases A and B. The case of the larger temperature perturbation contrasts sharply. In this case the stress collapse begins even before point $P$.

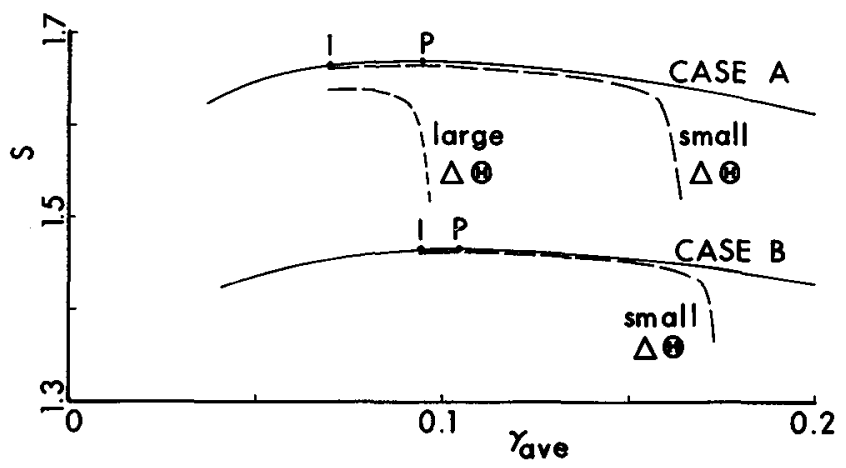
Fig. 3 - Stress vs, average strain (or time, since $Y_{\text {ave }}=1$ ). Homogeneous and
perturbed responses for cases $A$ and $B$.

Figures 4 and 5 show the evolution of temperature and plastic strain rate at the center and edge of the band in comparison to the homogeneous case. As is to be expected the behavior paraliels the stress response. At first the temperature and strain rate deviate slowly from the homogeneous response, but when the stress collapses, they both rise steeply in the center. At the edge the temperature levels off to a plateau while the plastic strain rate (not shown for the edge position) drops towards zero. At first it appears that the temperature and plastic strain rate change so as to compensate each other without appreciably affecting the stress response, but eventually thermal softening wins out in the center. At the edge the stress drops due to momentum transfer from the center and effectively quenches both the temperature rise and the plastic deformation.

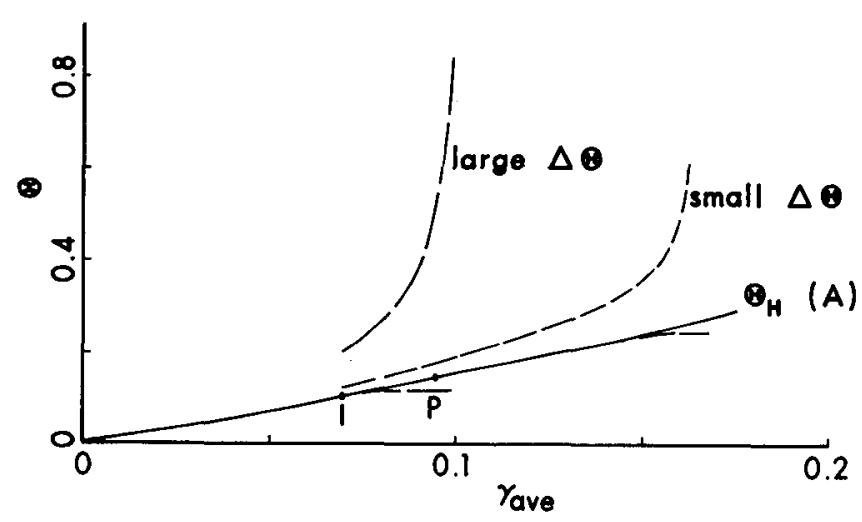

Fig. 4 - Temperature rise for case A. Upper curves are for the center of the band, lower curves for the edge. Solid curve is the homogeneous case. 


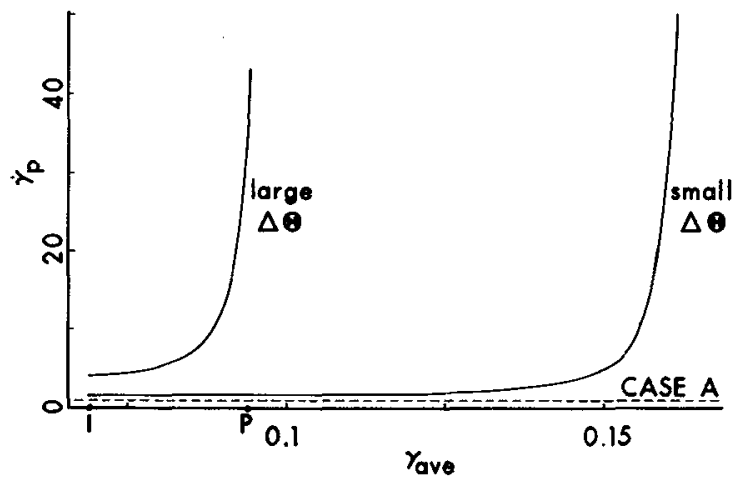

Fig. 5 - Plastic strain rate in the center of the band for case A. Reference rate (homogeneous deformation) is close to 1.

Figures 6 and 7 show cross sections of temperature and plastic strain rate for case A (with the larger temperature perturbation) at several times during the rapid

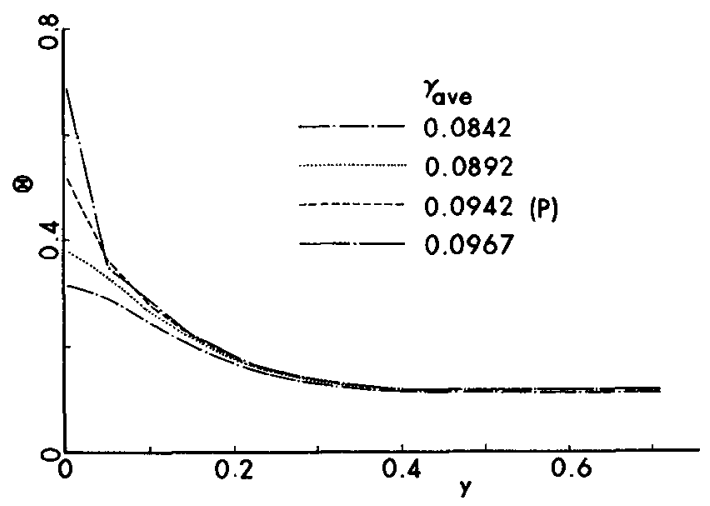

Fig. 6 - Cross sections of temperature rise at times indicated.

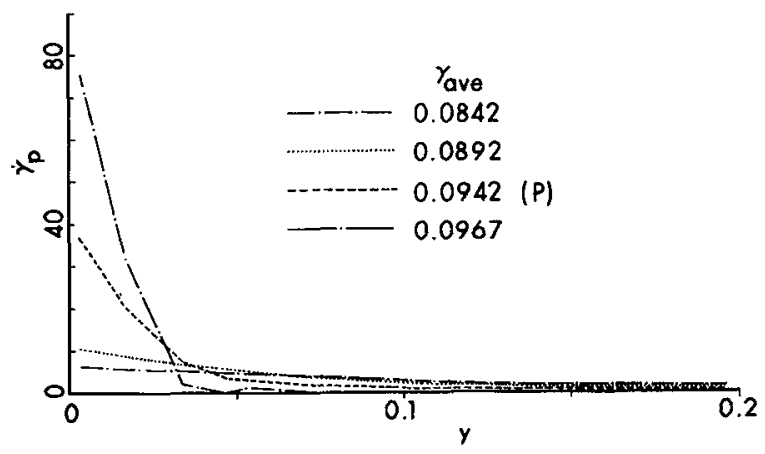

Fig. 7 - Cross sections of plastic strain rate at times indicated. 
transition. The most notable feature is the narrowing of the most active region of deformation. This is particularly evident in the cross sections of plastic strain rate. Note that for the latest time the plastic strain rate in the center of the band is nearly 80 times the average applied strain rate.

\section{III - MODIFICATIONS FOR A DIPOLAR EFFECT}

As a shear band forms, it is evident that very steep local strain gradients must develop. Therefore, it has seemed worthwhile to reformulate the problem in terms of a dipolar theory of plasticity. This has been accomplished in a straight forward manner by modifying the theory due to Green. McInnis, and Naghdi / $/$ / to include a rate effect. In a dipolar material it is assumed that, in addition to the usual traction forces that do work against velocities, there are hypertractions, denoted $\bar{\sigma}$ and having the dimensions of stress times length, that do work against velocity gradients. Decomposition of strain gradients into elastic and plastic parts introduces a new internal variable, denoted $\bar{d}_{p}$ in this paper, which requires its own evolutionary constitutive equation. As shown in $/ 9 /$, dipolar plasticity introduces length scales that are characteristic of the material itself. In this paper it is supposed that there is only one intrinsic length scale for elastic, plastic, or viscoplastic deformation, which is denoted $\bar{l}$. In addition to the nondimensional quantities introduced previous $7 y$, we require

$$
d_{p}=h \bar{d}_{p}, \quad \sigma=\bar{\sigma} / \bar{\ell}_{\kappa_{0}}, \quad l=\bar{l} / \mathrm{h} .
$$

The full set of equations with a Von Mises type flow condition now takes the form

$$
\begin{array}{ll}
\text { Momentum: } & \left(s-\ell \sigma,{ }_{y}\right){ }_{2}=\rho \dot{v} \\
\text { Energy: } & \dot{\theta}=k \theta, y y+s \dot{\gamma}_{p}+\sigma \dot{d}_{p}
\end{array}
$$

Constitutive Equations:

$$
\begin{aligned}
& s=\mu\left(v, y-\dot{\gamma}_{p}\right), \quad \dot{\gamma}_{p}=\Lambda s \\
& \dot{\sigma}=\ell \mu\left(v, y y-\dot{d}_{p}\right), \quad \dot{d}_{p}=\frac{\Lambda}{l} \sigma
\end{aligned}
$$

Reference Response and Work Hardening:

$$
\kappa=\left(1+\frac{\psi}{\psi_{0}}\right)^{n}, \dot{w}_{p}=\kappa \dot{\psi}=s \dot{\gamma}_{p}+\sigma \dot{d}_{p}
$$

Yield Condition:

$$
\left(s^{2}+\sigma^{2}\right)^{\frac{1}{2}}=(1-a \theta)\left\{1+b \Lambda\left(s^{2}+\sigma^{2}\right)^{\frac{1}{2}}\right\}_{K}^{m}
$$

In these equations $\Lambda$ plays the role of a plastic multiplier and is to be determined from yield condition when the loading point $(s, \sigma, \theta)$ lies outside the static yield surface, as determined by the last equation with $\Lambda$ set equal to zero. Extra boundary conditions are also required. These are chosen to be $\sigma(+1, t)=0$, so that the dipolar effect will effectively vanish outside the shear band.

Homogeneous solutions to these equations are exactly the same as before, but now the response to perturbations, computed for case $B$ with the smaller temperature bump, appears to be quite different, although long computational run times have not yet been achieved. Figure 8 shows the evolution of the central plastic strain rate for a nondimensional intrinsic length $\ell=10^{-2}$. The rate decays rapidly back toward the average applied rate, at least for short times after introduction of the perturbation, so that the dipolar effect appears to stabilize the deformation. 


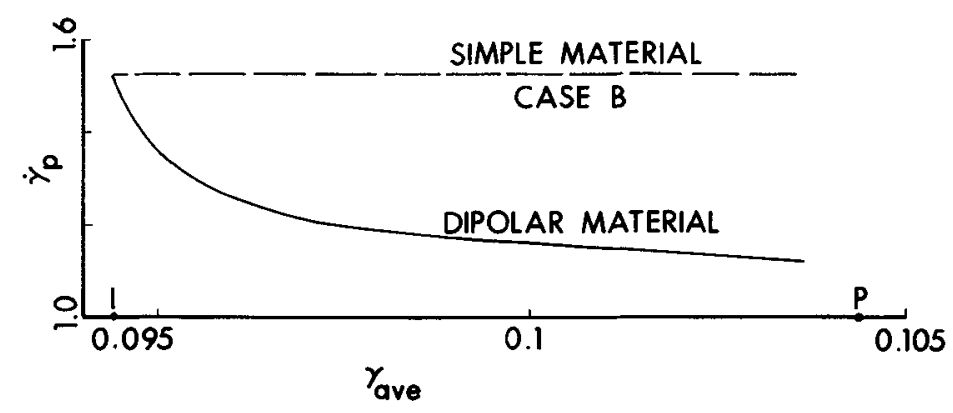

Fig. 3 - Plastic strain rate in the center of the band for case B at early times. Dipolar material compared to simple material.

\section{IV - DISCUSSION AND CONCLUSIONS}

The response to the small temperature perturbation is qualitatively very similar for both cases $A$ and $B$. Figure 3 shows that, although the stress levels are different due to the different viscous response, the shapes of the homogeneous respone curves are similar, and so are the shapes of the response to perturbation. For something on the order of 5 or $6 \%$ average strain past the peak of the homogeneous curve the stress path deviates oniy slightly until stress collapse sets in at the end of the calculation. Similar behavior was reported by Merzer /6/ who used a completely different flow law for viscoplasticity. Thus, it appears that stress collapse is inherent in the process of band formation with the details depending on the specific material model used.

The calculation with the larger perturbation in temperature for case $A$ shows that the details of stress collapse also depend strongly on the magnitude of the perturbation. Although case B has not been run with the larger temperature disturbance, there is no reason to expect that the results would be qualitatively different from case $A$.

The most striking aspect of the response patterns in Figure 3 is their similarity to those obtained in analyzing imperfection sensitivity for a bifurcation probiem, eg., see $/ 10 \%$. The onty thing missing is the bifurcation branch itself. Figures 4 and 5 for temperature and plastic strain rate reinforce this idea.

Although calculations for the dipolar case have not yet progressed far enough to be definitive, it appears that the effect, even for a very small inherent material length scale, will be highly stabilizing. This would be in accordance with the experience to date for elastic systems where, as in $/ 11 \%$, higher order effects allow smooth transitions instead of discontinuities.

Unfortunately, because of computational instabilities that set in, it has not yet been possible to continue calculations to the point where a final configuration for the shear band appears. The calculations reported in /2/ indicated that a peak value for plastic strain rate may occur, but reexamination has indicated numerical instability. The difficulty seems to be that eventually the extreme localization of the band defeats the ability of the present numerical method to resolve the details.

\section{REFERENCES}

/1/ Clifton, R. J., Duffy, J., Hartley, K. A. and Shawki, T. G., Scrip. Met. $\underline{18}$ (1984) 443. 
/2/ Wright, T. W. and Batra, R. C., Int. J. P7as. 1 (1985) in press.

/3/ Litonski, J., Bull. I'Acad. Poi. Sci. 25 (1977) 7.

/4/ Johnson, G. R., Hoegfelt, J. M., Lindholm, U. S. and Nagy, A., J. Eng. Mat. \& Tech., Trans. ASME 105 (1983) 42 and 105 (1983) 48.

/5/ Burns, T. J., Sandia Report SAND83-1901, Albuquerque, NM (1983).

16/ Merzer, A. M., J. Mech. Phys. So1. 30 (1982) 323.

/7/ Costin, L. S., Crisman, E. E., Hawley, R. H. and Duffy, J., in Mechanical

Properties at High Rates of Strain, 1979, Inst. Phys. 47 (1979) 90.

18/ Becker, E. B., Carey, G. F. and Oden, J. T., Finite Elements, An Introduction, Prentice-Hait (1981) Englewood Cliffs, NJ.

19/ Green, A. E., McInnis, B. C. and Naghdi, P. M., Int. J. Eng. Sci. 6 (1968) 373.

/10/ Iooss, G. and Joseph, O. D., Elementary Stability and Bifurcation Theory, Springer-Verlag (1980) New York - Heidelberg - BerTin.

/11/ Aifantis, E. C. and Serrin, J. B., J. Colloid and Interface Sci. 96 (1983) 517 and 96 (1983) 530. 